\title{
Color perception differentiates Alzheimer's Disease (AD) from Vascular Dementia (VaD) patients
}

Dr. Arnaoutoglou N.A. ${ }^{1}$, Assist. Prof. Arnaoutoglou M. ${ }^{2}$, Dr. Nemtsas P. ${ }^{2}$, Prof. Costa V. ${ }^{2}$, Prof. Emer. Baloyannis S.J. ${ }^{2}$, Prof. Ebmeier K.P. ${ }^{1}$

${ }^{1}$ Department of Psychiatry, University of Oxford, Oxford, UK

${ }^{2}$ First Department of Neurology, AHEPA Hospital, Aristotle University of Thessaloniki, Thessaloniki, Greece

Corresponding author:

Arnaoutoglou Nikitas

Department of Psychiatry, University of Oxford,

Warneford Hospital,

Warneford Ln,

Oxford OX3 $7 J X, U K$

Tel: + 44 (0)1865 223635

Fax: + 44 (0)1865 793101

Email: nikitas.arnaoutoglouepsych.ox.ac.uk 


\section{Abstract}

Background: Alzheimer's Disease (AD) and Vascular Dementia (VaD) are the most common causes of dementia in older people. Both diseases appear to have similar clinical symptoms, such as deficits in attention and executive function, but specific cognitive domains are affected. Current cohort studies have shown a close relationship between deposits and age-related macular degeneration (Johnson et al. 2002, Ratnayaka et al. 2015). Additionally, a close link between the thinning of the retinal nerve fiber (RNFL) and AD patients has been described, while it has been proposed that AD patients suffer from a nonspecific type of color blindness (Pache, et al. 2003).

Methods: Our study included 103 individuals divided into three groups: A healthy control group $(n=35), A D(n=32)$ according to DSM-IV-TR, NINCDS-ADRDA criteria and $\operatorname{VaD}(\mathrm{n}=36)$ based on ... DS-AIREN, as well as Magnetic Resonance Imaging (MRI) results. The severity of patient's cognitive impairment, was measured with the Mini Mental State Examination (MMSE) and was classified according to the Reisberg global deterioration scale (GDS). Visual perception was examined using the Ishihara plates: "Ishihara Color Vision Test - 38 Plate".

Results: The three groups were not statistically different for demographic data (age, gender and education). The Ishihara colour blindness test has a sensitivity of $80.6 \%$ and a specificity of $87.5 \%$ to discriminate $A D$ and $V a D$ patients when an optimal (32.5) cut-off value of performance is used. Conclusion: Ishihara Color Vision Test - 38 Plate is a promising potential method as an easy and not time-consuming screening test for the differential diagnosis of dementia between AD and VaD.

Keywords: Dementia, Alzheimer's Disease (AD), Vascular Dementia (VD), Neuropsychological testing, Screening 
Page 3 of 17 International Psychogeriatrics

Introduction

Dementia is a syndrome that is characterized by impairment of memory and at least one other cognitive domain (aphasia, apraxia, agnosia, executive function). The disorder mainly affects the elder and the published prevalence of dementia doubles with every 5-year increment in age (World Alzheimer Report, 2014). Worldwide prevalence is estimated at 47.5 million, is expected to double up by 2030 and triple by 2050. Alzheimer's Disease (AD) is the most common form of dementia in the elderly, accounting for 60 to 70 percent of cases (World Health Organization, 2015). A definitive diagnosis of AD requires post-mortem histopathologic examination, which is rarely done in life. Clinical criteria for the diagnosis of probable AD have been established by the National Institute of Neurological and Communicative Disorders and Stroke and the Alzheimer's Disease and Related Disorders Association (NINCDS-ADRDA) (MCKhann et al., 1984) and American Psychiatric Association (DSM-IV-TR) (American Psychiatric Association, $2000)$.

The second most common form of late onset dementia and the most common form of secondary dementia is vascular dementia (VaD). VaD is heterogeneous, due to different, small and large vessel related causes (Bowler, 2007). The National Institute of Neurological Disorders and Stroke and Association Internationale pour la Recherché et l'Enseignement en Neurosciences criteria (NINDS-AIREN) are most detailed and most widely used (Roman et al., 1993). One of the earliest clinical criteria for the differential diagnosis of VaD is the Hachinski Ischemic scale (HIS), which was established specifically to distinguish VaD from AD. Number of studies have shown that current clinical criteria do not separate AD from VaD patients with high specificity (Wetterling et al., 1996, Holmes et al., 1999, Wallin et al., 2003).

The current neurochemical background of AD is characterized by the reduction of essential neurotransmitters, such as acetylcholine, serotonin, noradrenaline and glutamate, within specific regions of the cortex and the hippocampus, with the concurrent neuropathological presence of amyloid plaques and neurofibrillary tangles. On the other hand, VaD is conceptualized by the aggregated impact of small and large strokes leading to brain cell death and overall neuron and axon loss (Jacoby et al., 2008).

Given that different pathological entities contribute to AD and VaD, different pharmacological treatments have been proposed (e.g. Acetylcholinesterase inhibitors are only effective in AD). Early intervention is highly desirable, since it is most likely to be effective, 
but requires differentiating patients with different risk profiles. Moreover, the identification and differentiation of prodromal stages of dementia into homogeneous groups of patients will facilitate new drug studies (Jack et al., 2011, European Medicines Agency, 2012, Dubois et al., 2014,). Importantly, it has been proposed that delaying the onset of dementia by 5 years without affecting mortality would reduce the population prevalence by 50\%, greatly reducing its impact on the general population (World Alzheimer Report, 2014 ). Several cognitive instruments have been evaluated for their ability to detect and differentiate early AD (Lonie et al., 2009, Thompson et al., 2010, Tan et al. 2015 ).

There are two different visual streams (parvocellular and magnocellular) that are involved in color perception (Livingstone and Hubel, 1987). Although aging has been related to the miosis and the yellowing of the lens, that may induce color perception disturbances (Verriest 1963), differences in performance are not consistently linked to age. Recent findings in AD patients suggest that there is a close link between macular degeneration and $\beta$-amyloid deposits in the retina (Ratnayaka et al., 2015), while others observed a thinning of

the RNFL and dysregulation of the magnocellular pathway (Parisi et al., 2001, Sartucci et al., 2010). The loss of visuospatial skills is an early feature of AD that is sometimes prominent at presentation (Mendez et al., 1990, Guerin et al., 2002) and the performance of AD patients in color discrimination tests is reduced both due to vision structures like the primary cortex and the retinal gaglionar cells (Salamone et al., 2009).

We investigated whether the performance of the visual perception between AD and VaD patients could improve the differential diagnosis for these two diseases. Interestingly, although there have been studies investigating the correlation between dementia and color blindness (Kaeser et al., 2015), the complete 38 plate version, one of the most popular color blindness test had never been evaluated. Thus, we decided to estimate the usefulness of the extended Ishihara Color Blindness test, as an easy and quick test for the differential diagnosis between $A D$ and $V a D$ in their early stages.

\section{Methods}

Measures and Procedure

Participants in the study were patients of the Memory clinic during the time period from 2007 to 2011, First Neurological Department, AHEPA University Hospital, Thessaloniki, Greece. The study adhered to the principles of the Declaration of Helsinki and the protocols employed had been approved by the Hospital's Research and Ethics Committee. All

Page 4 of 17 
participants provided informed consent before the evaluation. Demographic data (age, gender, level of education) were recorded and each participant underwent dementia evaluation, including a detailed clinical history, neurological examination, MRI, complete dementia blood screening test and EEG. Cognitive impairment was screened with the MMSE (Folstein et al., 1975) by the associate neuropsychologist of the memory clinic and patients were diagnosed by a board certified Neurologist (V.C.) and classified according to the Reisberg scale (Reisberg et al., 1982). Afterwards, all potential participants had a detailed ophthalmological exam to exclude patients with ocular pathology. Participants with MRI findings of large occipital ischemic lesions or ocular pathology and history of ocular disease, trauma, surgery, or laser treatment were excluded from the study. All participants used refractory corrections, appropriate for the viewing distances employed in the various tests. None suffered from any systemic or ocular conditions known to affect color vision. The inclusion criteria were met by 68 individuals: AD ( $n=32$, eleven males, mean age 73.3 years) according to DSM-IV-TR, NINCDS-ADRDA criteria and VaD ( $n=36$, twelve males, mean age 75.6 years) based on ...DS-AIREN, as well as MRI results. In addition, a healthy control group ( $\mathrm{n}=35,16$ males, mean age 74,2 years) was recruited.

\section{Visual perception test}

The most common test for screening for color blindness is the Ishihara Color deficiency test. It consists of 38 pseudoisochromaric plates (PIP). The PIP could represent a digit or a pathway. In addition, within the test there are plates that reveal figures or trail-tracing plates that are only identified by color deficient individuals. People with normal color vision can make up to two errors. Previous studies have proved that the cut-off value (CI 99\%) of errors in the general population is $>36$ while six errors suggest definitely that the participant suffers from color blindness (Verriest, 1982). Visual perception of all participants was evaluated with the use of Ishihara plates: "Ishihara Color Vision Test -38 Plate". The test was performed in a pleasant, quiet environment and the plane of the plate was adequately illuminated. Detailed instructions were given and the patients were asked to confirm their ability to recognize and detect basic colors, through a basic primary and secondary color wheel. A paper version of the 38 plate version of the Ishihara Test was performed.

\section{Statistics}

Statistical analysis was performed with SPSS (Scientific Package for Statistic Science Version 23 of SPSS Inc., USA). Analysis of variance (ANovA) was used to estimate any significant variation between the three groups within their age, MMSE, Reisberg Scale and 
total Ishihara score. In addition, stepwise multiple analysis, using the Scheffé's Alpha procedure, estimated the mean differences. Possible differences in the distribution of Gender and Education were calculated between all groups with Pearson's chi-squared test of significance (.2). Univariate ANOVA test was used, to compare the VaD with AD patient's performance in the Ishihara test, when controlling for Reisberg category, MMSE, Age and Education. A Receiver Operating Curve (ROC) analysis was used to estimate the discriminative validity of the Ishihara test as a diagnostic test between AD and VaD patients.

\section{Results}

At total of 171 individuals agreed to participate in our research. Sixty-eight $(\mathrm{M}: 43, \mathrm{~F}: 25)$ were excluded based on the selection criteria. Data on 103 participants diagnosed with $A D(n=32), \operatorname{VaD}(n=36)$ and healthy controls ( $\mathrm{n}=35)$ were analysed in the context of the present study. There was no difference in categorical variables (gender, level of education) between the three groups (Table 1). We were interested to assess total Ishihara score as a test for the differential diagnosis between the three groups. Analysis of variance (ANovA) was used to estimate any significant variation between the three groups for age, MMSE, Reisberg Scale and total Ishihara score (Table 2). This showed no significant differences in the age, but indicated differences in MMSE and Reisberg scale (as expected, since healthy controls were included) and importantly, total Ishihara scores. To further investigate this finding we applied a stepwise multiple analysis, using the Scheffé's Alpha procedure. The post hoc analysis with the Scheffé's method, showed differences in the MMSE and Reisberg scale between Controls and AD, as well as VaD patients, but not statistically significant differences between $A D$ and $V a D$ with these tests. Interestingly, Ishihara score was significantly different between AD and VaD participants. Based on this finding we performed a post-hoc ANovA comparing VaD with AD participants, controlling for Reisberg, MMSE, Gender, Age and Education, which gave us a significant difference in Ishihara scores between types of dementia $(F(1,61)=9.558, \mathrm{p}=0.003$; Table 3; Fig. 1). Finally, a RoC curve was used to estimate the discriminative power of the Ishihara test as diagnostic test (Fig. 2). The optimal cut-off value for discriminating AD from VaD participants was 32.50 with a sensitivity of $80.6 \%$ and specificity 87.5\%, $\mathrm{AUC}=0.819, \mathrm{SD}=0.59$ which results in a CI 95\% $=(0.703,0.934)(\mathrm{Fig} .2)$.

Discussion 
Page 7 of 17

\section{International Psychogeriatrics}

The World Health Organization and the G8 Dementia Summit have identified dementia and AD prevention as a major public health priority (World Health Organisation, 2012, G8 Dementia Summit Declaration, 2013). Early intervention to modifiable risk factors for $A D$ and $V a D$ is highly desirable, since it is most likely to be effective, but requires differentiating patients with different risk profiles. The US Food and Drug Administration (FDA) and the European Medicines Agency (EMA), is moving in the direction of developing new diagnostic criteria, that will facilitate the identification and differentiation of early stages of dementia into homogeneous groups of patients and will optimize new drug studies (Jack et al., 2011, European Medicines Agency, 2012, Dubois et al., 2014, ).

We wanted to develop a method to differentiate AD and VaD patients on clinical grounds, based on previous studies that were promising on that aspect (De Jager et al., 2003). Cronin-Colomb et al. suggested that there is a deficit in color discrimination in $A D$ that is specific to blue hues, and in the absence of such damage to anterior visual structures, peristriate and inferotemporal visual cortical neuropathological lesions are most likely the substrate for the deficit (Cronin-Golomb et al., 1993). A disruption of the visual signals mediated by area 17 and more specifically in the magnocellular or the M pathway of visual processing was supported by other studies (Hof and Morrison, 1990, Gilmore et al., 1994). Optic nerve and retinal ganglion cell degeneration is not a specific characteristic of AD according to Curcio et al. (Curcio and Drucker, 1993), while Davies et al. also, support that optic nerve degeneration is not a feature of $A D$ and the deficit result from cortical dysfunction (Davies et al., 1995). Independent of the pathophysiological basis, visual dysfunctions in AD has been recently studied as a predictor of early dementia, as well as the progression of the disease (Crutcher et al., 2009, Zola et al., 2013). One of the most difficult tasks in the assessment of vision in AD is the selection of the appropriate tool that will facilitate communication and perception of the test (Cronin-Golomb and Hof, 2004). We decided to use Ishihara plates, because the process of the test is easy to understand and indeed perception was confirmed by our patients. Additionally, it uses arithmetic (Arabic) digits, a common international code and can be applied worldwide. The test is short and patients can cooperate and remain focused. In contrast to sophisticated exams our method can be easily applied in an outpatient visit from specialized medical doctors and educated general practitioners that might have a key role in the accurate assessment and early diagnosis of dementia (Tang et al., 2016). Interestingly, our study revealed statistically significant differences between AD and VaD patients' performance in the Ishihara test, even in the earliest stages of dementia, which are the most important for pharmaceutical intervention (European Medicines Agency, 2012, Dubois et al., 
2014, Suri et al., 2014). Thus, one of the most important practical application of our findings is the use of Ishihara test in future cognitive battery tests, in order to facilitate earlier intervention in the prodromal stages of the diseases. To this aspect, Ishihara test will also facilitate differentiation of early stages of dementia into homogeneous groups of patients and will optimize new drug studies.

Methodological considerations

The usefulness of Ishihara plates in the differential diagnosis of AD and VaD does have limitations. Firstly, earlier studies have shown that the prevalence of congenital color blindness in Caucasians of European ancestry is estimated around 8\% in men and $0.4 \%$ in women (Birch 2012). The aforementioned prevalence vary depending on the origin of the participant, but still implies an important limitation to approximately 10\% of the population. Secondly, chronic illnesses, trauma, infarcts, lesions, medication or chemicals that affect the retina or the cerebrum, may be some of the causes of acquired color blindness. Furthermore, we had to exclude all participants suffering from any systemic or ocular conditions known to affect color vision, as well as patients with MRI findings of large occipital ischemic lesions. Another important limitation that should be taken under consideration is the small sample size of our research and the exclusion of the Hachinski scales from our analysis. Lastly, despite the ease of the process, cooperating with dementia patients is always a challenge and a pleasant, quiet and trusted environment must be secured.

Conclusion

Ishihara Color Vision Test - 38 Plate is a promising method as an easy and quick test for the differential diagnosis between AD and VaD. Further studies are required to confirm and extend our findings and determine the reliability and clinical usefulness of our method in other samples.

Declaration of Interest

None.

Description of Author Roles 
Dr. Arnaoutoglou, N.A. participated in setting the research questions, designing the study, carrying it out, analysing the data and the writing of the article. Assist. Prof. Arnaoutoglou, M. participated in setting the research questions, designing the study and the writing of the article. Dr. Nemtsas, P. participated in analysing the data and the writing of the article. Prof. Costa, V. participated in carrying it out, supervised the data collection. Prof. Em. Baloyannis, S.J. participated in formulating the research questions, designing the study. Prof. Ebmeier, K.P. participated in formulating the research questions, supervised the analysis of the data and the writing of the article

\section{Acknowledgments}

Dr. Arnaoutoglou N.A. was supported by the Greek Scholarship Foundation (4893), as part of a Ph.D. research project. The funders had no role in the design of the study, the collection, the analysis, and interpretation of data. 
International Psychogeriatrics

References

American Psychiatric Association (2000). Diagnostic and Statistical Manual of Mental Disorders (IV-TR). 4th edition-text revised. 4th ed. Washington, D.C

Bowler, J. V. (2007). Modern concept of vascular cognitive impairment. British Medical Bulletin, 83, 291-305.

Cronin-Golomb, A. \& Hof, P. R. (eds.) (2004). Interdisciplinary Topics in Gerontology and Geriatrics, Basel: Karger AG.

Cronin-Golomb, A., Sugiura, R., Corkin, S. \& Growdon, J. H. (1993). Incomplete achromatopsia in Alzheimer's Disease. Neurobiology of Aging, 14, 471-7.

Crutcher, M. D., Calhoun-Haney, R., Manzanares, C. M., Lah, J. J., Levey, A. I. \& Zola, S. M. (2009). Eye tracking during a visual paired comparison task as a predictor of early dementia. American Journal of Alzheimers Disease \& Other Dementias, 24, 258-66.

Curcio, C. A. \& Drucker, D. N. (1993). Retinal ganglion cells in Alzheimer's disease and aging. Annals of Neurology, 33, 248-57.

Davies, D. C., Mccoubrie, P., Mcdonald, B. \& Jobst, K. A. (1995). Myelinated axon number in the optic nerve is unaffected by Alzheimer's disease. British Journal of Ophthalmology, 79, 596-600.

De Jager, C. A., Hogervorst, E., Combrinck, M. \& Budge, M. M. (2003). Sensitivity and specificity of neuropsychological tests for mild cognitive impairment, vascular cognitive impairment and Alzheimer's disease. Psycholochical Medicine, 33, 103950.

Dubois, B. et. al. (2014). Advancing research diagnostic criteria for Alzheimer's disease: the IWG-2 criteria. Lancet Neurology, 13, 614-29.

European Medicines Agency (2012). Qualification opinion of Alzheimer's disease novel methodologies/biomarkers for: PET amyloid imaging (positive/negative) as a biomarker for enrichment, for use in regulatory clinical trials in predementia Alzheimer's disease Available:

http://www.ema.europa.eu/docs/en_GB/document_library/Regulatory_and_procedura 1_guideline/2012/04/WC500125018.p̄df [Accessed 16 February 2012].

Folstein, M. F., Folstein, S. E. \& Mchugh, P. R. (1975). "Mini-mental state". A practical method for grading the cognitive state of patients for the clinician. Journal Psychiatric Research, 12, 189-98.

G8 Dementia Summit Declaration. (2013). Global Action Against Dementia Available:

https://www.gov.uk/government/uploads/system/uploads/attachment_data/file/265869 /2901668 G8 DementiaSummitDeclaration acc.pdf [Accessed 11 December 2013].

Gilmore, G. C., Wenk, H. E., Naylor, L. A. \& Koss, E. (1994). Motion perception and Alzheimer's disease. Journal of Gerontology, 49, P52-7.

Guerin, F., Belleville, S. \& Ska, B. (2002). Characterization of visuoconstructional disabilities in patients with probable dementia of Alzheimer's type. Journal of Clinical Experimental Neuropsychology, 24, 1-17. 
Hof, P. R. \& Morrison, J. H. (1990). Quantitative analysis of a vulnerable subset of pyramidal neurons in Alzheimer's disease: II. Journal of Comparative Neurology and Psychology, 301, 55-64.

Holmes, C., Cairns, N., Lantos, P. \& Mann, A. (1999). Validity of current clinical criteria for Alzheimer's disease, vascular dementia and dementia with Lewy bodies. The British Journal of Psychiatry, 174, 45-50.

Page 10 of 17 
Jack, C. R., Jr., Albert, M. S., Knopman, D. S., Mckhann, G. M., Sperling, R. A., Carrillo, M. C., Thies, B. \& Phelps, C. H. (2011). Introduction to the recommendations from the National Institute on Aging-Alzheimer's Association workgroups on diagnostic guidelines for Alzheimer's disease. Alzheimers Dement, 7 , 257-62.

Jacoby, R., Oppenheimer, C., Dening, T., Thomas, A., (2008). "Oxford Textbook of Old Age Psychiatry.", OUP Oxford; 4Rev Ed edition, ISBN-10: 0199298106, 86-90

Johnson, L. V., Leitner,WP., Rivest, AJ., Staples, MK., Radeke, MJ., Anderson, DH. (2002). The Alzheimer's A.-peptide is deposited at sites of complement activation in pathologic deposits associated with aging and age-related macular degeneration. Proceedings of the National Academy of Sciences of the United States of America, 99(18), 11830-11835.

Kaeser, PF, Ghika, J., Borruat, F. (2015). Visual signs and symptoms in patients with the visual variant of Alzheimer disease. BMC Ophthalmology, 15 (1).

Livingstone, M. S. and Hubel, D. H. (1987). Psychophysical evidence for separate channels for the perception of form, color, movement, and depth. Journal of Neuroscience $7(11), 3416-3468$.

Lonie, J. A., Tierney, K. M., Herrmann, L. L., Donaghey, C., O'carroll, R. E., Lee, A. \& Ebmeier, K. P. (2009). Dual task performance in early Alzheimer's disease, amnestic mild cognitive impairment and depression. Psychological Medicine, 39, 23-31.

Mckhann, G., Drachman, D., Folstein, M., Katzman, R., Price, D. \& Stadlan, E. M. (1984). Clinical diagnosis of Alzheimer's disease: report of the NINCDS-ADRDA Work Group under the auspices of Department of Health and Human Services Task Force on Alzheimer's Disease. Neurology, 34, 939-44.

Mendez, M. F., Mendez, M. A., Martin, R., Smyth, K. A. \& Whitehouse, P. J. (1990). Complex visual disturbances in Alzheimer's disease. Neurology, 40, 43943.

Pache M., Smeets, CH., Gasio, PF., Savaskan, E., Flammer, J., Wirz-Justice, A., Kaiser, HJ. (2003). Colour vision deficiencies in Alzheimer's disease. Age and Ageing, $32(4): 422-426$.

Parisi, V., Restuccia, R., Fattapposta, F., Mina, C., Bucci, MG, Pierelli, F. (2001). Morphological and functional retinal impairment in Alzheimer's disease patients. Clinical Neurophysiology 112(10), 1860-1867.

Ratnayaka, J. A., Serpell, L., Lotery, A. (2015). Dementia of the eye: The role of amyloid beta in retinal degeneration. Eye 29(8), 1013-1026.

Reisberg, B., Ferris, S. H., De Leon, M. J. \& Crook, T. (1982). The Global Deterioration scale for assessment of primary degenerative dementia. American Journal of Psychiatry, 139, 1136-9.

Roman, G. C., Tatemichi, T. K. et al. (1993). Vascular dementia: diagnostic criteria for research studies. Report of the NINDS-AIREN International Workshop. Neurology, 43, 250-60. 
Salamone, G., Di Lorenzo, C., Mosti, S., Lupo, F., Cravello, L., Palmer, K., Musicco, M., Caltagirone, C. (2009). Color discrimination performance in patients with alzheimer's disease. Dementia and Geriatric Cognitive Disorders $27(6), 501-507$.

Sartucci, F., Borghetti, D., Bocci, T., Murri, L., Orsini, P., Porciatti, V., Origlia, N., Domenici, L. (2010). Dysfunction of the magnocellular stream in Alzheimer's disease evaluated by pattern electroretinograms and visual evoked potentials. Brain Research Bulletin 82(3-4), 169-176.

Suri, S., Topiwala, A., Mackay, C. E., Ebmeier, K. P. \& Filippini, N. (2014). Using 
International Psychogeriatrics Page 12 of 17

structural and diffusion magnetic resonance imaging to differentiate the dementias. Current Neurology and Neuroscience Reports, 14, 475.

Tan, L. P., Herrmann, N., Mainland, B. J. \& Shulman, K. (2015). Can clock drawing differentiate Alzheimer's disease from other dementias? International Psychogeriatrics, 27, 1649-60.

Tang, E. Y., Birdi, R. \& Robinson, L. (2016). Attitudes to diagnosis and management in dementia care: views of future general practitioners.

International Psychogeriatrics, 1-6.

Thompson, C., Brodaty, H., Trollor, J. \& Sachdev, P. (2010). Behavioral and psychological symptoms associated with dementia subtype and severity. International Psychogeriatrics, 22, 300-5.

Verriest, G. (1963). Further studies on acquired deficiency of color discrimination. Journal of the Optical Society of America, 53, 185-195.

Verriest, G. (1982) Colour Vision Deficiencies VI -Page 161 ISBN 978-90-6193729-6

Wallin, A., Milos, V., Sjogren, M., Pantoni, L. \& Erkinjuntti, T. (2003). Classification and subtypes of vascular dementia. International Psychogeriatrics, 15 Suppl 1, 27-37.

Wetterling, T., Kanitz, R. D. \& Borgis, K. J. (1996). Comparison of different diagnostic criteria for vascular dementia (ADDTC, DSM-IV, ICD-10, NINDS-AIREN). Stroke, 27, 30-6.

World Alzheimer Report. (2014). Dementia and Risk Reduction Available: https://www.alz.co.uk/research/WorldAlzheimerReport2014.pdf [Accessed October 2014 ]

World Health Organisation. (2012). Dementia: a public health priority Available: http://www.who.int/mental_health/publications/dementia_report_2012/en/ [Accessed 2012].

World Health Organization. (2015). Dementia Fact Sheet. Available: http://www.who.int/mediacentre/factsheets/fs362/en/ [Accessed April 2016].

Zola, S. M., Manzanares, C. M., Clopton, P., Lah, J. J. \& Levey, A. I. (2013). A behavioral task predicts conversion to mild cognitive impairment and Alzheimer's disease. American Journal of Alzheimer's Disease \& Other Dementias, 28, $179-84$. 
Fig. 1: Box plots of standardized residuals of Ishihara score, controlling for age, education, MMSE and the Reisberg Scale for both patients groups. Fig. 1 $150 \times 120 \mathrm{~mm}(300 \times 300 \mathrm{DPI})$

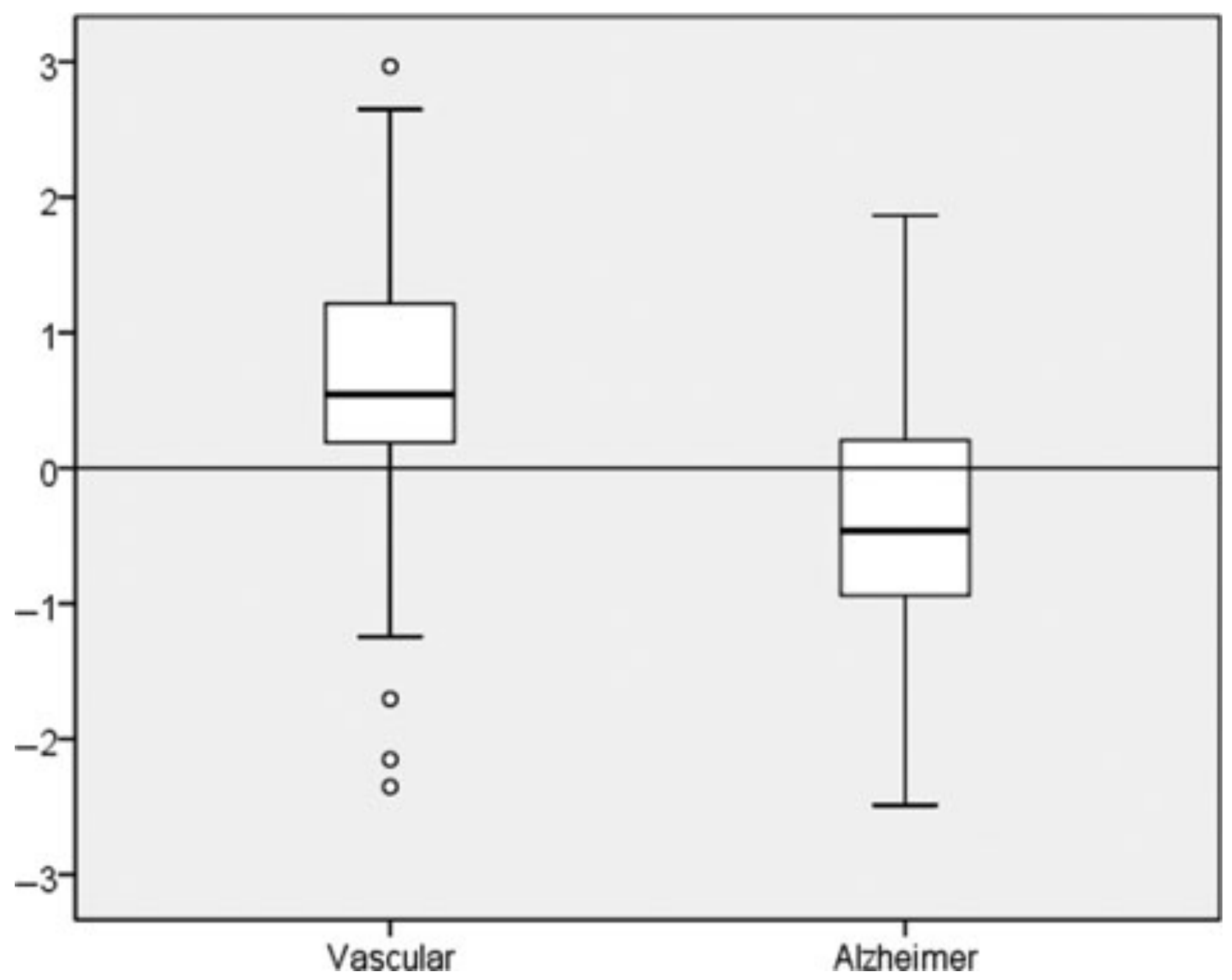


Fig. 2 ROC showing sensitivity and 1-specificity of unadjusted Ishihara scores in discriminating $\mathrm{AD}$ from VaD(A); data points (B) Fig. 2 266x107mm (300 x 300 DPI )

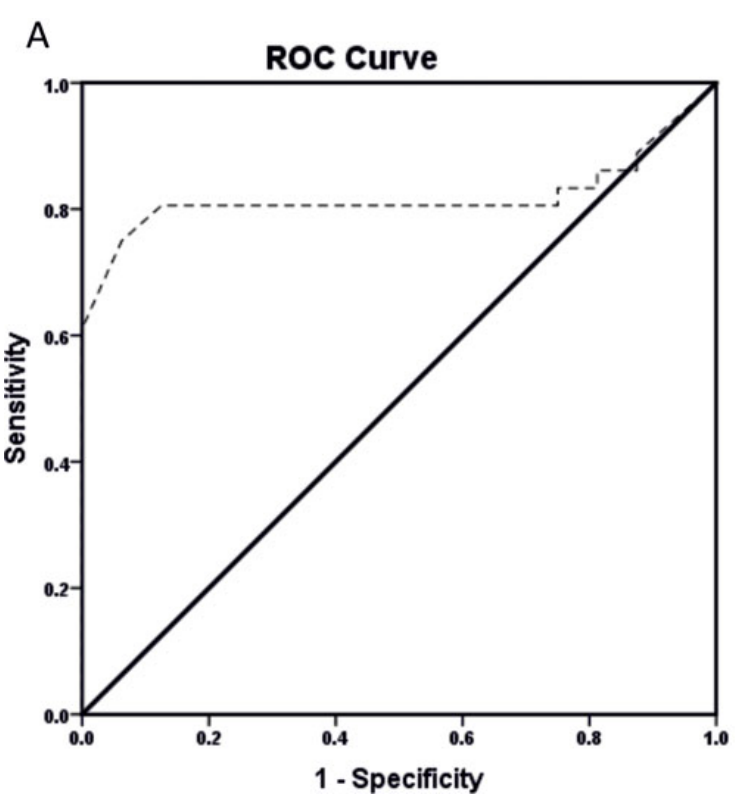

B

\begin{tabular}{|c|c|c|c|c|c|c|c|c|}
\hline \multicolumn{9}{|l|}{ Cut-scores } \\
\hline Equal or Less & Sens & Spec & PPV & NPV & TP & $\mathrm{FP}$ & $\mathrm{TN}$ & $\mathrm{FN}$ \\
\hline 25.5 & 0.81 & 0.38 & 0.53 & 0.69 & 26 & 23 & 14 & 6 \\
\hline 27.5 & 0.81 & 0.44 & 0.56 & 0.72 & 26 & 20 & 16 & 6 \\
\hline 28.5 & 0.81 & 0.47 & 0.57 & 0.73 & 26 & 19 & 17 & 6 \\
\hline 29.5 & 0.81 & 0.53 & 0.60 & 0.75 & 26 & 17 & 19 & 6 \\
\hline 30.5 & 0.81 & 0.63 & 0.66 & 0.78 & 26 & 14 & 23 & 6 \\
\hline 31.5 & 0.81 & 0.81 & 0.79 & 0.82 & 26 & 7 & 29 & 6 \\
\hline 32.5 & 0.81 & 0.88 & 0.85 & 0.84 & 26 & 5 & 32 & 6 \\
\hline 33.5 & 0.78 & 0.91 & 0.88 & 0.82 & 25 & 3 & 33 & 7 \\
\hline 34.5 & 0.75 & 0.94 & 0.91 & 0.81 & 24 & 2 & 34 & 8 \\
\hline 35.5 & 0.61 & 1 & 1.00 & 0.74 & 20 & 0 & 36 & 12 \\
\hline 36.5 & 0.53 & 1 & 1.00 & 0.70 & 17 & 0 & 36 & 15 \\
\hline 37.5 & 0.28 & 1 & 1.00 & 0.61 & 9 & 0 & 36 & 23 \\
\hline \multicolumn{9}{|c|}{ Abbreviations: Sens, sensitivity; Spec, specificity; PPV, Positive Predictive Value; } \\
\hline \multicolumn{9}{|c|}{ NPV, Negative Predictive Value; TP, True Positive; FP, False Positive; } \\
\hline \multicolumn{9}{|c|}{ TN, True Negative; FN, False Negative; } \\
\hline
\end{tabular}

https://mc.manuscriptcentral.com/ipg 
Page 15 of 17 International Psychogeriatrics

Table 1: Pearson's chi-square test (X2) for the distribution of gender and education between the three groups

\begin{tabular}{|c|c|c|c|c|c|}
\hline & Variables & Control & $A D$ & $\operatorname{VaD}$ & P value \\
\hline \multirow[t]{2}{*}{ Gender } & MALE & 16 & 11 & 12 & 0.50 \\
\hline & FEMALE & 19 & 21 & 24 & \\
\hline \multirow[t]{5}{*}{ Education } & BAS IC & 14 & 9 & 12 & 0.42 \\
\hline & EDUCATION & & & & \\
\hline & INTERMEDIATE & 11 & 17 & 13 & \\
\hline & EDUCATION & & & & \\
\hline & $\mathrm{HIGH}$ & 10 & 6 & 11 & \\
\hline
\end{tabular}


International Psychogeriatrics Page 16 of 17

Table 2: ANOVA and post-hoc Scheffé method of the three study groups

\begin{tabular}{llllll}
\multicolumn{2}{c}{ Controls } & \multicolumn{2}{c}{ VD } & \\
\cline { 1 - 2 } Mean SD & Mean & SD & Mean & SD & $\begin{array}{l}\text { Post-hoc } \\
\text { Scheffé }\end{array}$
\end{tabular}

\begin{tabular}{llllllllll}
\hline Age & 74.2 & \pm 6.5 & 73.3 & \pm 5.8 & 75.6 & \pm 4.5 & 0.25 & $\mathrm{C}, \mathrm{A}, \mathrm{V}$ \\
MMSE & 29.2 & \pm 1.0 & 18.5 & \pm 6.1 & 19.8 & \pm 1.0 & 0.00 & $\mathrm{C}>\mathrm{V}, \mathrm{A}$ \\
Reisberg & 1 & \pm 0 & 4.1 & \pm 0.3 & 3.9 & \pm 1.7 & 0.00 & $\mathrm{C}<\mathrm{V}, \mathrm{A}$ \\
Ishihara & 37.7 & \pm 0.6 & 24.4 & \pm 1.7 & 31.3 & \pm 11.3 & 0.00 & $\mathrm{C}>\mathrm{V}>\mathrm{A}$
\end{tabular}


Page 17 of 17 International Psychogeriatrics

Table 3: Univariate ANOVA of the Ishihara test performance between AD and VaD patients, controlling for Diagnosis, Reisberg, MMSE, Gender, Age and Education.

Alzheimer vs. Vascular Dementia

Dependent Variable: Ishihara Score

\begin{tabular}{llll}
\hline Source & df & F & Sig. \\
\hline Corrected Model & 6 & 21.842 & 0.000 \\
Intercept & 1 & 0.246 & 0.622 \\
REISBERG & 1 & 0.117 & 0.733 \\
MMSE & 1 & 5.823 & 0.019 \\
GENDER & 1 & 0.651 & 0.423 \\
AGE & 1 & 0.176 & 0.676 \\
EDUCATION & 1 & 0.361 & 0.550 \\
DIAGNOSIS & 1 & $\mathbf{9 . 5 5 8}$ & $\mathbf{0 . 0 0 3}$ \\
Error & 61 & & \\
Total & 68 & & \\
Corrected Total & 67 & & \\
R-Squared $=0.682$ & (Adjusted R-Squared $=0.651)$ &
\end{tabular}

